\title{
Rheological Properties of Natural Fiber Reinforced PP Composites
}

\author{
Xianyi Shao ${ }^{1, \mathrm{a}}$, Luju He $\mathrm{H}^{2, \mathrm{~b}^{*}}$, Li Ma ${ }^{3, \mathrm{c}}$ \\ ${ }^{1}$ College of Physics \& Electronic Engineering, Taizhou University, Taizhou 318000, China \\ ${ }^{2}$ Education and Information Technology Center, Guangdong University of Petrochemical \\ Technology, Maoming 525000, Guangdong, China \\ ${ }^{3}$ College of Mechanical and Electrical Engineering, Guangdong University of Petrochemical \\ Technology, Maoming 525000, Guangdong, China \\ asxy8718@163.com, bheluju@gdupt.edu.cn, 'mali@gdupt.edu.cn
}

Keywords: Natural fiber; Surface modification; Rheological properties; Composite Abstract. Cellulose (CE), sawdust (SD) and wheat straw (WS) were subjected to triethoxysilane (AS) and methacriloxy propyl trimethoxy silane (MS), and maleic anhydride grafted polypropylene (MAPP) to improve interfacial bonding with matrix. PP/fiber composites were prepared in a rheomixer equipped with two rotor blades and adjustable temperature, mixing rate and mixing time. Torque values of each composite formulation were recorded with respect to time to determine changes in rheological properties of composites. It was found that increase in fiber loading increases stabilization torque of composites. The extent of increase at $40 \mathrm{wt} \% \mathrm{CE}$ loading was about $11 \%$. Silane treatments increased stabilization torque values up to $1 \mathrm{wt} \%$ silane treatment with respect to fiber weight, irrespective of the fiber employed due to increased interactions between fiber and matrix. MAPP treatment decreased stabilization torque due to plasticizing effect of MAPP.

\section{Introduction}

The composite materials in polymer matrix reinforced with natural fibers are receiving more attention in both academia and various industries. The mechanical properties of a natural fiber-polymer matrix composite are controlled to a large extent by the efficiency of the bonding at the fiber-matrix interfacial boundary. The principal function of the interface is to facilitate the transfer of stress from fiber to fiber, across the matrix. Cellulose fiber is known to exhibit poor bonding characteristics with a polymeric matrix ${ }^{[1]}$. This is mainly due to the presence of organic and inorganic substances on the fiber surface, and the hydrophilic nature of cellulose, which prevents effective adhesion between the fiber surface and the polymer matrix. The surface of natural fiber such as sisal fiber is usually not suitable for creating a strong bond with a polymeric matrix. The bond strength has been successfully improved by modifying the fiber surface with either chemical or physical surface treatment such as heat treatment, corona treatment, plasma treatment, silanization and acetylation ${ }^{[2-5]}$.

In this work, three natural fibers, namely cellulose (CE), sawdust (SD) and wheat straw (WS) were used as reinforcement for PP matrix. Three coupling agents, (3-aminopropyl)-triethoxysilane (AS), methacriloxy propyl trimethoxy silane (MS), and maleic anhydride grafted polypropylene (MAPP) were employed in order to enhance interfacial interactions between hydrophilic fiber and hydrophobic matrix. Effect of fiber type and loading and treatment type on stabilization torque was studied in order to determine relative rheological behavior of the composites.

\section{Experimental}

Cellulose (CE), sawdust (SD) and wheat straw (WS) with fiber content of 10, 20, 30 and 40 wt $\%$ were treated with triethoxysilane (AS), methacriloxy propyl trimethoxy silane (MS), and maleic anhydride grafted polypropylene (MAPP), and then Chopped dried short untreated or treated fibers and PP pellets were compounded by using XSS-300 Rheomixer equipped with two rotor blades rotating in opposite directions. There were three walls surrounding the chamber where filler and matrix melt. 
Temperature of the three walls could be adjusted separately for optimum temperature control. The composites were prepared at mixing temperature of $458 \mathrm{~K}$, rotor speed of $50 \mathrm{rpm}$ and mixing time of 10 minutes. The composites were prepared with CE, SD and WS at 10, 2030 and $40 \mathrm{wt} \%$ of fiber loadings. Composites were heated without pressure for 4 minutes to $458 \mathrm{~K}$ in order to avoid void formation and then pressed at $10 \mathrm{MPa}$ pressure at the same temperature for 6 minutes. The specimens were cooled to $313 \mathrm{~K}$ in 6 minutes. During compounding, torque vs time data of the mix can be damped through "Convert Data" software program to determine rheological response of the composites. Effect of fiber type and loading and treatment type on stabilization torque was analyzed in order to determine relative rheological behavior of the composites.

\section{Results and discussion}

A typical Torque vs. time data for cellulose/PP composites is shown in Fig.1. The initial torque increased rapidly by incorporation of polymer which is depicted as a peak at around 40 seconds. Peak heights decreased with increasing filler loading because polymer loading decreased with increasing filler loading. Torque decreased rapidly as soon as temperature of polypropylene increased and melting occurred. After complete melting at around 90 seconds, cellulose was fed to rheomixer which was accompanied by an increase in viscosity. This second peak was proportional to the fiber loading. Wetting of the fibers by the polymer and dispersion decreased torque up to a stable value that is called stabilization torque. Composite reached stabilization torque at around 400 seconds. A stable torque is also an indicator of homogenization of filler in the melt ${ }^{[6]}$. When stabilization values were compared, it was clearly seen that an increase in fiber loading was accompanied by an increase in stabilization torque. Stabilization torque was $4.3 \mathrm{~N} \cdot \mathrm{m}$ for neat PP whereas $9.06 \mathrm{~N} \cdot \mathrm{m}$ for $40 \mathrm{wt} \%$ cellulose filled PP which corresponds to a $111 \%$ increase. This result can be treated as an increase in viscosity with increasing fiber loading. Fig. 2 illustrates variation of stabilization torque with respect to fiber loading for PP/CE composites. Surface treatment was employed to promote fiber dispersion and to enhance interfacial bonding between fiber and matrix. Frequently, surface modification of the fiber results in a reduction in shear viscosity relative to untreated material, which may be explained by reduced interaction between the filler and dispersion medium, although a decreased tendency towards filler network formation may also be a contributory factor. If present in polymer phase, these chemicals may also exert a lubricating effect causing a reduction in viscosity. In our stabilization torque data, all surface treatments have decreased stabilization torque, especially at low fiber loadings. There are two interactions in a filled polymer system; Fiber-fiber interactions and fiber-matrix interactions. Silane coupling agents increase polarity and hydrophobicity of CE fibers, which yields to decreased particle-particle interactions. That is, agglomeration or network formation is decreased that would have a decreasing effect on viscosity. On the other hand, particle-matrix interaction is increased due to enhanced matrix-polymer adhesion. This would have a positive effect on viscosity. As seen in Fig.2, stabilization torque significantly decreased with AS and MS treatment of $\mathrm{CE}$ at 10 and 20wt\% CE loadings, but stabilization torque was almost the same at 30 and $40 \%$ loadings. These results reveal that at lower fiber loadings, the dominating interaction is particle-particle interaction, but at higher loadings, particle-matrix interactions begin to dominate. MAPP treatment always had a negative effect on stabilization torque, which is more pronounced for low fiber loadings.

Stabilization torque of neat PP and 10wt\%MAPP treated PP were compared in Fig.3. It can be seen that $10 \mathrm{wt} \%$ MAPP incorporation into PP decreases torque from 4.30 to 3.36 corresponding to a $22 \%$ decrease which proves that decrease in viscosity of composites is due to interaction of MAPP with PP. Fig.4-6 illustrate effect of treatment type, concentration and fiber type on stabilization torque at 30wt\% loading for AS, MS and MAPP treated PP/CE composites, respectively. Stabilization torque were measured for 3 different silane concentrations $(0.5,1,2.5 \mathrm{wt} \%$ of fiber $)$. As observed from the figures, silane treatment leads to increase in stabilization torque values. The increase is maximum at $1 \mathrm{wt} \%$ silane treated composites. In all silane treated composites, stabilization torque values decreases at $2.5 \mathrm{wt} \%$ silane concentration regardless of fiber type. When AS and MS treated composites were 
compared, AS treatment provided higher stabilization torque values compared to MS treatment because particle-particle interactions are more pronounced in AS treatment. These results suggest that matrix-fiber interaction or adhesion between fiber and matrix reached maximum at $1 \%$ silane treatment. It can also be concluded that $2.5 \%$ silane treatment would have formed a layer on the fibers which can be attributed to unreacted silanes or long chain siloxanes on fiber surface. This layer would restrict interaction between fiber and matrix decreasing stabilization torque.

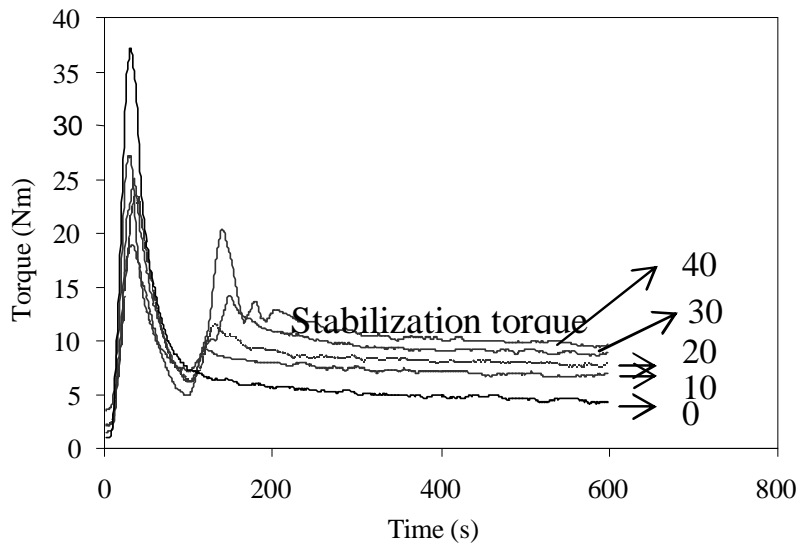

Fig.1. Torque vs time data for $0,10,20,30$, $40 \mathrm{wt} \%$ cellulose loaded PP composites

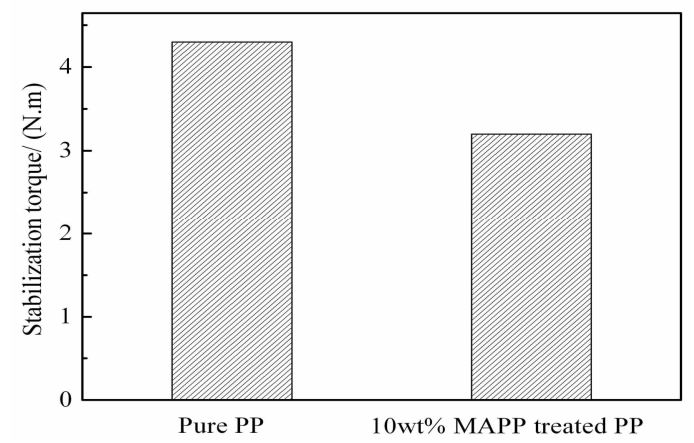

Fig.3. Comparison of stabilization torque of pure and MAPP treated PP

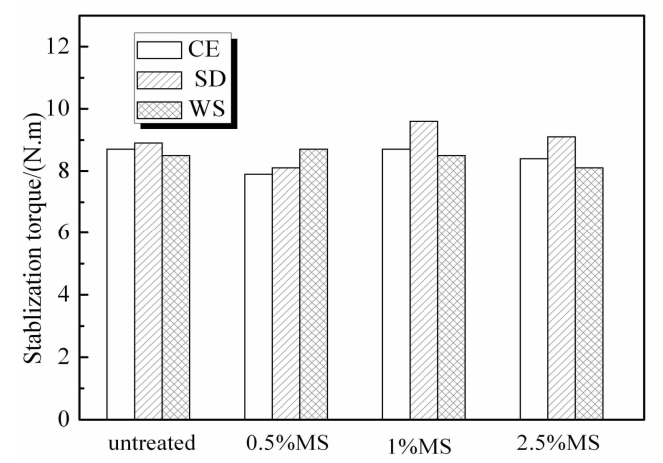

Fig.5. Effect of concentration of MS treatment on stabilization torque of $30 \mathrm{wt} \%$ CE, SD and WS loaded PP composites

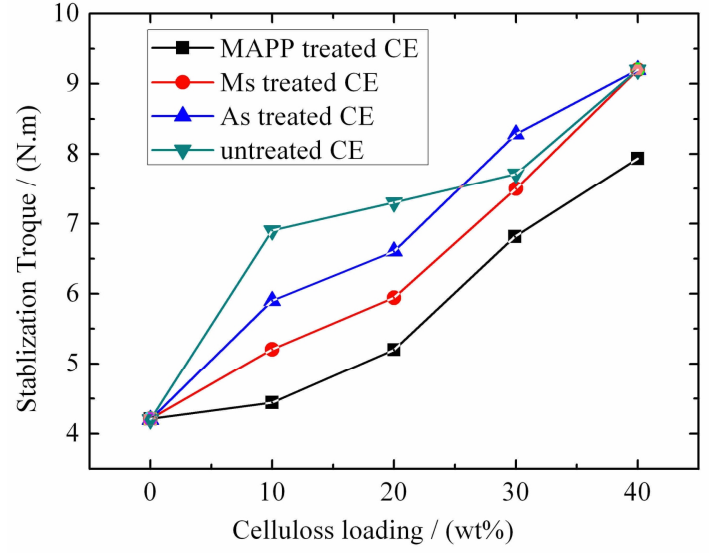

Fig.2. Variation of stabilization torque with respect to cellulose loading and treatment

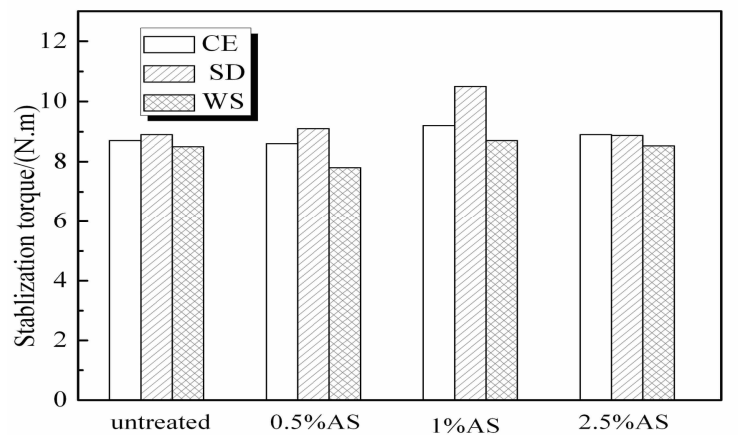

Fig.4. Effect of concentration of AS treatment on stabilization torque of $30 \mathrm{wt} \% \mathrm{CE}, \mathrm{SD}$ and WS loaded PP composites

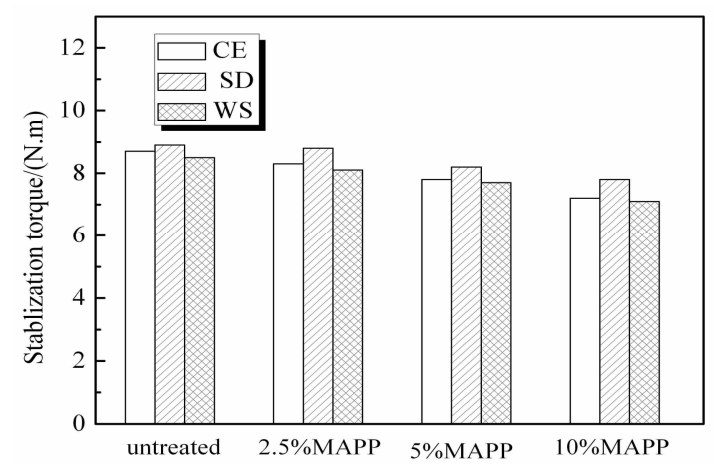

Fig.6. Effect of concentration of MAPP treatment on stabilization torque of 30wt $\%$ CE, SD and WS loaded PP composites

Viscosity of suspension of rigid spherical particles can be characterized by Eisenstein's equation which holds for rigid particles in dilute concentrations as seen in Eq. ${ }^{[7]}$.

$$
\eta / \eta_{1}=\left(1+k E \phi^{2}\right)
$$


Where $\eta$ is the viscosity of suspension, $\eta_{1}$ is the viscosity of suspending liquid (PP in our case), $k E$ is the Eisenstein coefficient (2.5 for dispersed spheres) and $\phi^{2}$ is the volume fraction of particles. Taking relative torque values as the viscosity of $\mathrm{CE} / \mathrm{PP}$ composite since shear rate and temperature was constant, experimental viscosities with changing filler volume fraction was compared with theoretical calculations according to Eisenstein's equation for PP/CE composites, as shown in Fig.7, deviation of torque from theoretical value is high, especially at high volume fractions. Treatment of CE decreased deviation from theoretical value at low filler loading. At high volume fractions, the model does not predict experimental values well as a result of particle-particle interactions. Increase in volume fractions increased particle-particle interactions, thus deviation from Eisenstein's equation was increased. It was also noted that silane treatment decreased deviation from the model because of decreased interactions between particles. The decrease in interactions would be treated as an evidence of enhanced dispersion of fillers in the matrix via silane treatment, especially at low volume fractions.

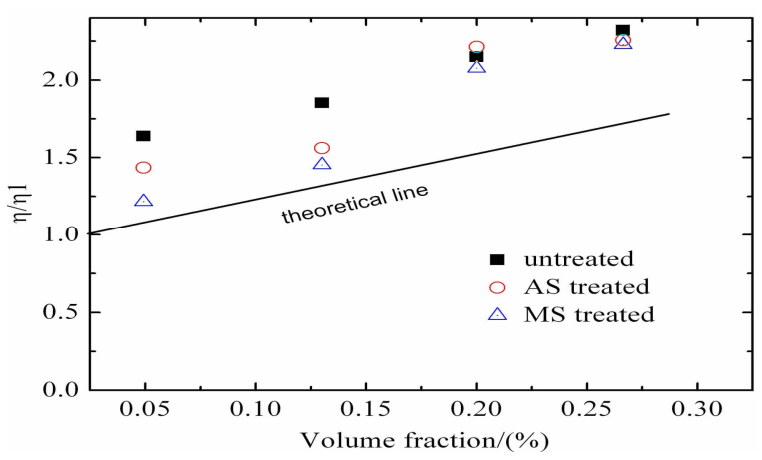

Fig.7. Variation of torque with respect to $\mathrm{CE}$ volume fraction for untreated and AS and MS treated CE.

\section{Conclusions}

Three natural fibers of cellulose (CE), sawdust (SD) and wheat straw (WS) were used as reinforcement for PP matrix. Three coupling agents, (3-aminopropyl)-triethoxysilane (AS), methacriloxy propyl trimethoxy silane (MS), and maleic anhydride grafted polypropylene (MAPP) were employed in order to enhance interfacial interactions between hydrophilic fiber and hydrophobic matrix. Torque data, which was a measure of rheological properties of composites, revealed that incorporation of fibers into PP increased stabilization torque of the composites. The extent of increase at $40 \mathrm{wt} \% \mathrm{CE}$ loading was about $11 \%$. Silane treatments increased stabilization torque values up to $1 \mathrm{wt} \%$ silane treatment with respect to fiber weight, irrespective of the fiber employed due to increased interactions between fiber and matrix. MAPP treatment decreased stabilization torque due to plasticizing effect of MAPP.

\section{Acknowledgements}

This work was financially supported by Taizhou Science \& Technology Project (Grant No. 14GY02) and Maoming Science \& Technology Project (Grant No. 915325).

\section{References}

[1] A. Ramzy, D. Beermann, L. Steuernagel, D. Meiners: Composites Part B Vol. 66 (2014), p.287

[2] G.A. Holt, P. Chow, J.D. Wanjura: Industrial Crops and Products Vol. 52 (2014), p. 627

[3] A. Pizzi, R. Kueny, F. Lecoanet, et.al: Industrial Crops and Products Vol.30 (2009), p. 235

[4] R.H. Bradley, I.L. Clackson, D.E. Sykes: Applied Surface Science Vol. 72 (1993), p. 143

[5] D. García-García, A. Carbonell, M.D. Samper: Composites Part B Vol. 78 (2015), p. 256

[6] P.V.Joseph, K.Koseph, S.Thomas: Composite Science and Technology Vol. 59 (1999), p. 1625

[7] M.N. Ichazo, C. Albano, J. Gonzales, R. Perera: Composite Structures Vol.54 (2001), p. 207 